\title{
Progressive Multifocal Leukoencephalopathy with Balanced CD4/CD8 T-Cell Infiltration and Good Response to Mefloquine Treatment
}

\author{
Nobuo Sanjo ${ }^{1}$, Satoko Kina ${ }^{1}$, Yukiko Shishido-Hara ${ }^{2}$, Yurie Nose ${ }^{1}$, Satoru Ishibashi ${ }^{1}$, \\ Tetsuya Fukuda ${ }^{3}$, Taketoshi Maehara ${ }^{4}$, Yoshinobu Eishi ${ }^{5}$, \\ Hidehiro Mizusawa ${ }^{1,6}$ and Takanori Yokota ${ }^{1}$
}

\begin{abstract}
A 53-year-old man was admitted for sub-acute progressive dementia and Gerstmann syndrome. MRI demonstrated lesions in the white matter involving the left parietal lobe, accompanied by speckled or faint linear peripheral enhancement. Brain biopsy revealed JC virus infection in oligodendrocytes and balanced infiltration of CD8+ and CD4+ T lymphocytes. We diagnosed progressive multifocal leukoencephalopathy (PML) with controlled inflammation. The finding of CD4/CD8 T cells in the infected PML brain suggested therapeutically valuable immune system involvement, which we decided to preserve by withholding corticosteroids. We treated the patient with risperidone, cytarabine and mefloquine to suppress virus replication, but not with the corticosteroid that is conventionally used in inflammatory PML cases. The patient was discharged three months after admission, and one year later, his score on the Mini-Mental State Examination had recovered to $26 / 30$, from $5 / 30$ on admission.
\end{abstract}

Key words: mefloquine, JC virus, progressive multifocal leukoencephalopathy, IRIS, PML

(Intern Med 55: 1631-1635, 2016)

(DOI: 10.2169/internalmedicine.55.6051)

\section{Introduction}

Progressive multifocal leukoencephalopathy (PML) is a fatal, demyelinating disease induced by opportunistic JC polyomavirus (JCV) infection of the central nervous system (CNS). PML has previously been diagnosed in patients with HIV or hematologic malignancy (1). The neuropathological characteristics of classical PML are demyelination, bizarre astrocytes and oligodendroglial nuclear inclusions. This profile has recently been modified by immunohistochemistry against viral capsid proteins (1-3), allowing detection of the virus within the nuclei of infected oligodendrocytes and astrocytes. Importantly, lymphocytic or plasma cell infiltration has proven to be rare in most cases (4). The absence of edema, mass effect and contrast enhancement on T1weighted magnetic resonance imaging (MRI) indicates the absence of inflammation in the brain $(5,6)$. The demonstration of JCV in the cerebrospinal fluid (CSF) by polymerase chain reaction (PCR) has become important for diagnosis, and the reach of the PCR technique has been advancing apace (7). Although antiretroviral therapy (highly active anti-retroviral therapy: HAART) has improved the treatment effect in HIV patients with PML, this therapy has sometimes been associated with aggravated clinical features due to the emergence of immoderate inflammation in PML lesions (8). Recently, in the wake of the increasing use of monoclonal antibodies as therapy for collagen diseases and

\footnotetext{
${ }^{1}$ Department of Neurology and Neurological Science, Tokyo Medical and Dental University, Japan, ${ }^{2}$ Department of Anatomic Pathology, Tokyo Medical University, Japan, ${ }^{3}$ Department of Haematology, Tokyo Medical and Dental University, Japan, ${ }^{4}$ Department of Functional Neurosurgery, Tokyo Medical and Dental University, Japan, ${ }^{5}$ Division of Surgical Pathology, Tokyo Medical and Dental University Hospital, Japan and ${ }^{6}$ National Centre for Neurology and Psychiatry, National Centre Hospital, Japan

Received for publication June 24, 2015; Accepted for publication August 27, 2015
}

Correspondence to Dr. Nobuo Sanjo, n-sanjo.nuro@tmd.ac.jp 
multiple sclerosis, PML has been diagnosed as an adverse effect associated with these agents (1). About half of those cases had lesions characterized by contrast enhancement on neuroimaging (9). Such PML cases accompanied by an improving clinical course and worsening of MRI lesions are called inflammatory PML (8), PML-immune reconstitution inflammatory syndrome (IRIS) (10) or other terms. Postmortem histopathological examination of a fatal case demonstrated severe inflammation with marked infiltration by macrophages and CD8+ T lymphocytes (11). However, the scope for further differential diagnosis among cases of JCVrelated leukoencephalopathy accompanied by lymphocytic inflammation is unclear. In this report, we present a PML patient with lymphocytic inflammation, which was demonstrated to be immunologically balanced, and discuss the importance of distinguishing between balanced and unbalanced inflammation in PML.

\section{Case Report}

A 53-year-old man presenting with rapid progressive cognitive impairment and right hemispatial neglect was admitted to our hospital. The patient had been treated once with chemotherapy and radiation for clinical stage IIB follicular lymphoma eight years previously at the Department of Hematology in our hospital, and had been successfully induced into the stage of stable disease. Computed tomography had revealed a small, swollen lymph node in his abdomen four years previously. The hematologist diagnosed a minor relapse and started continuous observation. Diffusion-weighted (DW) MRI images of the brain one month before his admission (Figure A) demonstrated high-intensity areas with internal low-intensity areas in the white matter of the left frontal, left parieto-temporal and right parietal lobes (Supplementary Material), which were observed as diffuse hyperintense lesions in T2-weighted images (Figure B). The lesions were hypointense in post-contrast T1-weighted images, with some tiny, speckled or faint linear enhancements at the edges (Figure C). At the time of admission, the patient did not receive any immunosuppressive therapy. A neurological examination revealed Gerstmann syndrome, memory impairment and right hemispatial agnosia. Peripheral blood tests showed no abnormality. Testing for HIV was negative. CSF examination demonstrated a cell count of 1 cell/ $\mu \mathrm{L}$ and total protein of $57 \mathrm{mg} / \mathrm{dL}$. Repeated PCRs were negative for JCV DNA in the CSF.

Brain biopsy was performed to distinguish between lymphoma relapse and PML. Examination of involved tissue at low magnification revealed the central area of the demyelinating lesion (Figure D), which was associated with infiltration by numerous lymphocytes and foamy histiocytes. Blood vessels proliferated in the area surrounding the lesion, and lymphoid cells were clustered in the perivascular spaces (Figure E). Observation at higher magnification revealed oligodendroglia-like cells with swollen nuclei of various sizes, many of which showed intranuclear inclusions of dot- shaped structures (Figure F, arrows). Immunohistochemistry specific to JC virus capsid proteins (VP2/3) (1) detected many cells harboring dot-shaped nuclear inclusions (Figure $\mathrm{G}$, arrow) and a few nuclei with full inclusions (FigureG, arrowhead).

To characterize the patient's brain inflammation, we performed immunohistochemistry using anti-CD3 (Nichirei, Tokyo, Japan), anti-CD4 (Nichirei), anti-CD8 (Nichirei), antiCD20 (Clone L26; Dako, Glostrup, Denmark) and antiCD68 antibodies (Clone PG-M1). The area surrounding the demyelinating lesion showed pronounced T-cell infiltration as well as numerous macrophages. Most fatal cases show predominant infiltration by $\mathrm{CD} 8+\mathrm{T}$ cells that overwhelm the CD4+ $\mathrm{T}$ cells $(6,8)$. However, intriguingly in this case, both $\mathrm{CD} 8+$ and $\mathrm{CD} 4+\mathrm{T}$ cells were infiltrating around the JC virus-infected cells, and the ratio of CD4/CD8 was 1.41 (Figure H, I). The ratio of CD4/CD8 in the cerebrospinal fluid was 1.73 . This probably indicated that the inflammatory reaction against the infectious agent was balanced. This differs from the fatal PML found in patients with a selfdestructive immune reaction or IRIS, in which autoclasialike uncontrolled proliferation of CD8+ T cells occurs (11). No atypical CD20+ cells were seen, even in areas of abundant CD3+ cells. CD68+ cells were mostly detected in the demyelinated areas of the white matter, and fragments positive for Kluver-Barrera staining were detected in the cytoplasm of these cells (data not shown), indicating the presence of phagocytized myelin sheaths from lytic oligodendroglia. We diagnosed the patient as having PML with balanced inflammation resulting from the anti-viral immune response (for details, see Table). We finally decided not to treat the patient with corticosteroid, which is widely recommended for use in cases of PML with inflammation or IRIS. After diagnosis (13 days after admission, about 1.5 months after onset), we started treating the patient with $1 \mathrm{mg} /$ day risperidone, a 5-HT2A receptor blocker (12), combined with $4 \mathrm{mg} / \mathrm{kg} /$ day cytarabine. Two weeks later, about two months after onset, we initiated mefloquine (Mephaquin; Hisamitsu Pharmaceutical, Tosu, Japan) at a dose of $275 \mathrm{mg} /$ day orally for three days, followed by $275 \mathrm{mg}$ once per week (12). Treatment with mefloquine was approved by our hospital Ethics Committee. We obtained written, informed consent from the patient. During this treatment with mefloquine, the patient did not complain of any symptoms associated with its potential adverse effects. After starting the mefloquine, his cognitive function gradually improved and, one month after initiation, his score on the Mini-Mental State Examination (MMSE) had recovered from 5/30 to 17/30. Brain MRI two months after admission demonstrated the absence of any lesion expansion and the disappearance of the gadolinium enhancement. The patient was discharged three months after admission. We maintained the treatment with mefloquine until one year after discharge, at which time the MMSE score had improved to 26/30. The patient's cognitive function has remained stable six months after the termination of mefloquine. 

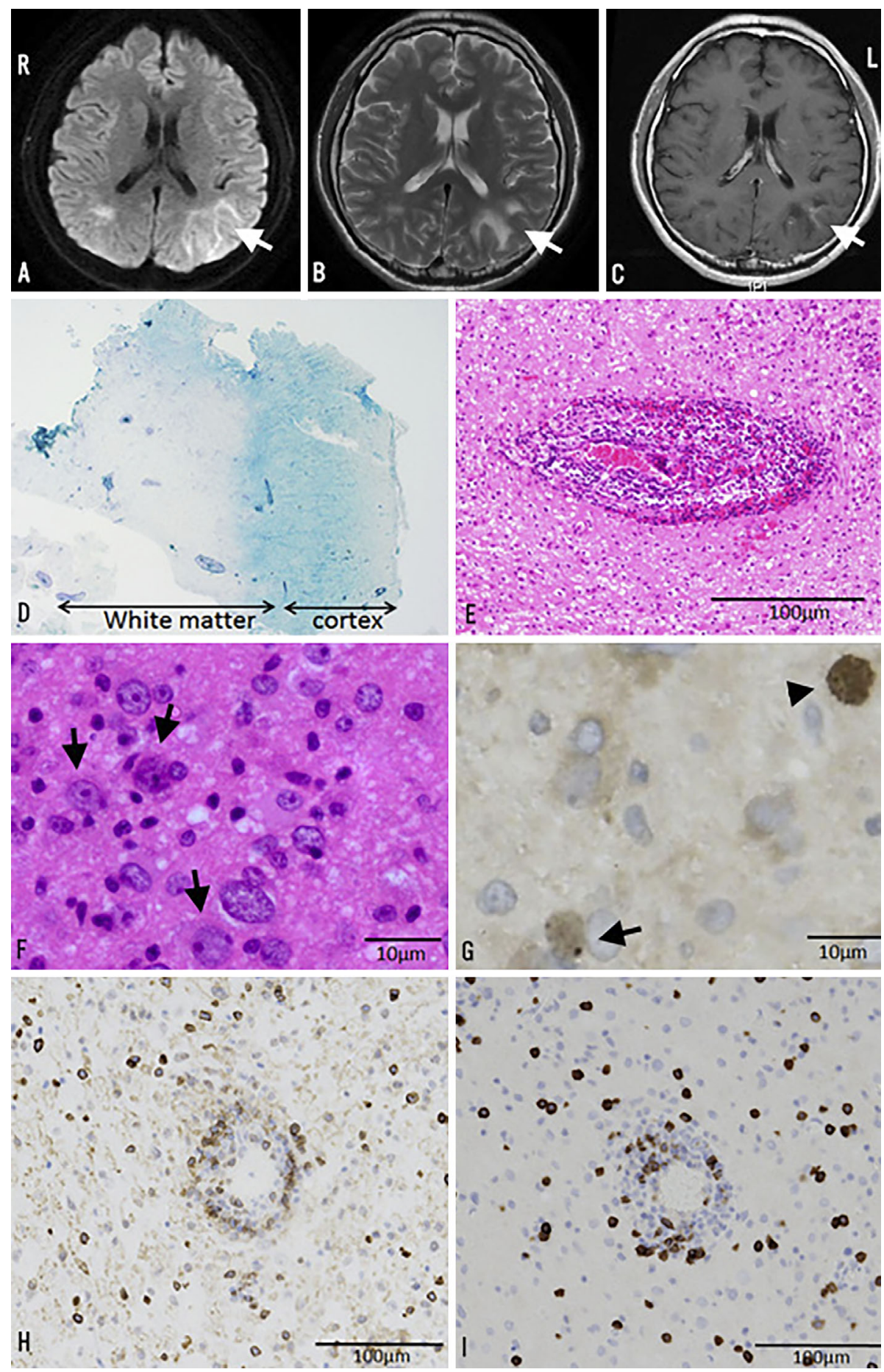

Figure. Axial DW (A) and T2-weighted (B) MR images demonstrating high-intensity lesions in parietal white matter bilaterally (arrows), with speckled or faint linear peripheral enhancement visible at the lesion edges in post-contrast T1-weighted MRI (C; arrow). Biopsied brain tissues show demyelination in white matter (D; Kluver-Barrera staining). Infiltration of lymphoid cells is apparent in the brain parenchyma as well as in the perivascular spaces $(E)$. Enlarged nuclei containing dot-shaped structures (arrows) are visible (F). Viral capsid proteins VP2/VP3 visualized by immunohistochemistry show punctate patterns (dot-shaped inclusions; arrow) or an even distribution throughout the nucleoplasm (full inclusions; arrowhead) $(G)$. CD4+ T cells $(H)$ and $C D 8+T$ cells $(I)$ are present at a relatively well-balanced ratio $(\mathrm{CD} 4 / \mathrm{CD8}=1.41)$. $\mathrm{R}$ : right, $\mathrm{L}$ : left

\section{Discussion}

The patient in this case was not in any apparent immuno- suppressive state at the onset of the PML, although he had received chemotherapy for follicular lymphoma eight years previously. PML may occur in those without apparent immunosuppression (13), but how it develops under such con- 
Table. Classification of PML by Inflammation Pattern.

\begin{tabular}{|c|c|c|c|}
\hline & classic PML & Fatal Inflammatory PML* & PML with balanced inflammation \\
\hline \multicolumn{4}{|l|}{ MRI } \\
\hline Brain oedema & - & + to ++ & \pm to + \\
\hline $\begin{array}{l}\text { Peripheral Gd enhancement at the } \\
\text { lesion }\end{array}$ & - & Speckles and/or linear & Speckles and/or linear \\
\hline \multicolumn{4}{|l|}{ Brain pathology } \\
\hline $\mathrm{T}$ cell profile & No infiltration & CD8 predominant & $\mathrm{CD} 4>=\mathrm{CD} 8$ \\
\hline Inclusions in infected oligodendrocyte & Dotlike to full inclusion & Dotlike to full inclusion & Dotlike predominant \\
\hline \multicolumn{4}{|l|}{ Treatment } \\
\hline Steroid & Not recommend & Recommend & Not recommend \\
\hline Mefloquine & Recommend & Recommend & Recommend \\
\hline
\end{tabular}

ditions remains obscure. It is possible that viremia and viral mutation occurred when the patient was treated with chemical agents including corticosteroid, and that these mutated JC viruses had slowly infiltrated into the brain. It is also possible that the virus had already entered the brain and had proliferated to cause the disease.

Although many guidelines recommend its use for PML, the efficacy of mefloquine is still controversial. This agent was originally used as an anti-malarial drug, but was found to have inhibitory activity against JC viral DNA replication in vitro in 2008 (14). The drug is assumed to be efficacious for PML when maintained at therapeutic concentrations, as in the brains of patients treated with approved doses (14). In our case, the cognitive impairment steadily recovered after starting mefloquine and the patient was eventually able to return to his job. Compared with other patients with PML, whether accompanied by inflammation or not, a dramatic benefit was achieved for this patient.

It was difficult for us to make the decision to refrain from using corticosteroid because most guidelines recommend corticosteroids for patients with PML accompanied by Gdenhancing lesions. Retrospectively, it seemed to be important for the efficacy of mefloquine and the prognosis of the patient to maintain competent immune responses against JCV infection in the CNS. We had formed a hypothesis to this effect based on the pathological finding that the ratio of CD4/CD8 indicated that the inflammatory situation in this case was under the control of a regulated infectious immune response. Importantly, the findings also showed that the inflammatory mechanism differed from that in PML with fatal excessive inflammation, in which $\mathrm{CD} 8+\mathrm{T}$ cells usually show destructively uncontrolled proliferation (Table) (4). Mefloquine itself does not actively remove JCV, but prevents replication of its DNA (14). Therefore, anti-JCV activity that cooperates with a well-controlled immune reaction should be crucial in removing the virus from the CNS. Further studies are necessary to clarify whether the inflammatory reactions in PML are crucial, and also whether treatment should be modified according to the patient's immune response profile, such as the ratio of $\mathrm{CD} 8$ to $\mathrm{CD} 4$, the $\mathrm{JCV}$ DNA levels in the CSF and the presence of MDR1 polymorphisms (15).
In conclusion, it is important to bear in mind that some PML patients are without apparent immunosuppression and that these patients probably have a good prognosis. Moreover, we recommend brain biopsy for evaluation of the $\mathrm{CD}$ 4/CD8 ratio of the $\mathrm{T}$ cells in the specimen before making a therapeutic decision for PML patients showing cerebral white matter lesions with peripheral gadolinium enhancement on MRI because corticosteroid use could cause inferior outcomes if the inflammation is under the control of a regulated infectious immune system.

\section{The authors state that they have no Conflict of Interest (COI).}

Treatment with mefloquine was approved by our hospital Ethics Committee. We obtained written, informed consent from the patient.

\section{Acknowledgement}

We thank Drs. Kazuo Nakamichi and Masayuki Saijo (Department of Virology I, National Institute of Infectious Diseases, Tokyo, Japan) for the analysis of JCV in the CSF.

\section{References}

1. Shishido-Hara Y. Progressive multifocal leukoencephalopathy and promyelocytic leukemia nuclear bodies: a review of clinical, neuropathological, and virological aspects of JC virus-induced demyelinating disease. Acta Neuropathol 120: 403-417, 2010.

2. Berger JR, Aksamit AJ, Clifford DB, et al. PML diagnostic criteria: consensus statement from the AAN Neuroinfectious Disease Section. Neurology 80: 1430-1438, 2013.

3. Aksamit AJ, Sever JL, Major EO, et al. Progressive multifocal leukoencephalopathy: JC virus detection by in situ hybridization compared with immunohistochemistry. Neurology 36: 499-504, 1986.

4. Gray F, Bazille C, Adle-Biassette H, Mikol J, Moulignier A, Scaravilli F. Central nervous system immune reconstitution disease in acquired immunodeficiency syndrome patients receiving highly active antiretroviral treatment. J Neurovirol 11 (Suppl 3): 16-22, 2005.

5. Shah R, Bag AK, Chapman PR, Curé JK. Imaging manifestations of progressive multifocal leukoencephalopathy. Clin Radiol $\mathbf{6 5}$ : 431-439, 2010.

6. Tan CS, Koralnik IJ. Progressive multifocal leukoencephalopathy and other disorders caused by JC virus: clinical features and pathogenesis. Lancet Neurol 9: 425-437, 2010. 
7. Nakamichi K, Tajima S, Lim CK, Saijo M. High-resolution melting analysis for mutation scanning in the non-coding control region of JC polyomavirus from patients with progressive multifocal leukoencephalopathy. Arch Virol 159: 1687-1696, 2014.

8. Huang D, Cossoy M, Li M, et al. Inflammatory progressive multifocal leukoencephalopathy in human immunodeficiency virusnegative patients. Ann Neurol 62: 34-39, 2007.

9. Clifford DB, De Luca A, Simpson DM, Arendt G, Giovannoni G, Nath A. Natalizumab-associated progressive multifocal leukoencephalopathy in patients with multiple sclerosis: lessons from 28 cases. Lancet Neurol 9: 438-446, 2010.

10. Weissert R. Progressive multifocal leukoencephalopathy. J Neuroimmunol 231: 73-77, 2011.

11. Metz I, Radue EW, Oterino A, et al. Pathology of immune reconstitution inflammatory syndrome in multiple sclerosis with natalizumab-associated progressive multifocal leukoencephalopa- thy. Acta Neuropathol 123: 235-245, 2012.

12. Kobayashi Z, Akaza M, Numasawa Y, et al. Failure of mefloquine therapy in progressive multifocal leukoencephalopathy: Report of two Japanese patients without human immunodeficiency virus infection. J Neurol Sci 324: 190-194, 2013.

13. Chang Y-Y, Lan MY, Peng CH, Wu HS, Chang D, Liu JS. Progressive multifocal leukoencephalopathy in an immunocompetent taiwanese patient. J Formos Med Assoc 106 (2 Supplement): S60S64, 2007.

14. Brickelmaier M, Lugovskoy A, Kartikeyan R, et al. Identification and characterization of mefloquine efficacy against JC virus in vitro. Antimicrobial agents and chemotherapy 53: 1840-1849, 2009.

15. Lee Nevin R. Neuropharmacokinetic heterogeneity of mefloquine in the treatment of progressive multifocal leukoencephalopathy. Intern Med 51: 2257, 2012.

(C) 2016 The Japanese Society of Internal Medicine http://www.naika.or.jp/imonline/index.html 